\title{
Using a Syndromic Surveillance System to Evaluate the Impact of a Change in Alcohol Law
}

\author{
Julia A. Dilley*1, Atar Baer², Jeff Duchin² and Julie E. Maher ${ }^{1}$ \\ 'Program Design \& Evaluation Services, Multnomah County Health Department and Oregon Public Health Division, Portland, OR, \\ USA; ${ }^{2}$ Public Health - Seattle \& King County, Seattle, WA, USA
}

\section{Objective}

To determine whether there were changes in alcohol-related emergency department (ED) visits in Washington State associated with statewide alcohol system deregulation.

\section{Introduction}

In November 2011, Washington State voters passed Initiative 1183 (I-1183) which closed state-owned and contracted liquor stores and opened the market for "hard liquor" sales in the private sector. The change in law was implemented on June 1, 2012. Increases in alcoholrelated ED visits were postulated as one potential impact if there was increased alcohol use or excessive consumption associated with the change in law.

\section{Methods}

We examined weekly counts of ED visits for alcohol-related conditions from all but one hospital in King County, Washington, for the period January 2010 - September 2013 (16 months after implementation of the law). King County includes approximately one-third of the state population and ED data are provided to the county as part of ongoing syndromic surveillance. Outcomes were ED visits with an alcohol-related chief complaint or diagnosis code. Chief complaint search strings included terms such as: alcohol, drunk, names of alcohol types (e.g., beer, whiskey, vodka), alcohol on breath. Diagnosis codes included ICD-9 codes previously described by the Centers for Disease Control and Prevention (CDC) for acute impacts of excessive alcohol consumption (see http://apps.nccd.cdc. gov/DACH_ARDI/Info/ICDCodes.aspx). We used a linear regression model with a spline at June 1, 2012, to determine if implementation of I-1183 was associated with changes in weekly ED visits. We fitted a series of regression models, stratified by both age groups $(<21,21-39,40+)$ and gender. The main covariates of interest were a binary "policy" coefficient with values of "0" prior to June 2012 and "1" including and after June 2012, and an interaction term (policy coefficient $\mathrm{x}$ week). The dependent variables in the regression models were log-transformed to achieve homogeneity in the error structure. The model also included covariates for background trend (time variable for week), seasonality (calendar quarters), and general healthcare access (total ED visits each week for any reason). Our models took into account autocorrelated error terms by using an autoregressive modeling approach with Newey-West standard errors and a lag of 12 in Stata v. 13.0.

\section{Results}

Statistically significant $(\mathrm{p}<.05)$ increases in alcohol-related ED visits post-law were observed among minors (age <21) and adults ages 40 and older. The initial increases among minors, however, appeared to decline over time. No significant changes in alcoholrelated ED visits were observed among adults ages 21-39. Findings may be limited by ecological bias as well as the choice of search terms included in the alcohol syndrome definition. Future validation of the syndrome definition may improve the sensitivity/specificity of the results. Both alcohol-related ED visits as a percentage of total visits and also counts of alcohol-related ED visits (with adjustment for total visits) were independently explored as the outcomes of interest. Patterns of significance were similar. Trend analysis must incorporate adjustment for known data quality issues in syndromic surveillance, such as data drop-offs or new hospitals being added during the evaluation period.

\section{Conclusions}

Alcohol-related ED visits increased among minors (age younger than 21) and adults ages 40 and older after changes in Washington State law increased availability of "hard liquor." Syndromic surveillance data were useful for describing public health impact of a change in law.

\section{Keywords}

Alcohol; Emergency department data; Public Health Law

\section{Acknowledgments}

This study was supported by Public Health Law Research, a national program of the Robert Wood Johnson Foundation.

\footnotetext{
*Julia A. Dilley

E-mail: julia.dilley@state.or.us
} 